\title{
Enhancing skin penetration of epigallocatechin gallate by modifying partition coefficient using reverse micelle method by Noorma Rosita
}

Submission date: 09-Oct-2019 11:10AM (UTC+0800)

Submission ID: 1189065259

File name: modifying_partition_coefficient_using_reverse_micelle_method.pdf (1.39M)

Word count: 5802

Character count: 30905 


\title{
Therapeutic Delivery
}

\section{Enhancing skin penetration of epigallocatechin gallate by modifying partition coefficient using reverse micelle method}

\author{
Noorma Rosita ${ }^{1}$, Vinta Arnike Meitasari ${ }^{1}$, Mirna Candra Rianti ${ }^{1}$, Dewi Melani Hariyadi' \& \\ Andang Miatmoko*,1 (iD \\ ${ }^{1}$ Department of Pharmaceutics, Faculty of Pharmacy, Universitas Airlangga, Kampus C Mulyorejo, Surabaya 60115, Indonesia \\ *Author for correspondence: andang-m@ff.unair.ac.id
}

\begin{abstract}
Aim: (-)-Epigallocatechin gallate (EGCG) has been reported as inducing apoptosis in cervical cancer. However, EGCG demonstrates low skin permeability. In order to develop topical delivery of EGCG, the partition coefficient, $\log$ P, was modified using a reverse 7 icelle method. Results \& methodology: During this study, Twe 780 and Span 80 were added to EGCG at hydrophilic-lipophilic balance (HLB) values of 4.3, 6 and 8 . The results showed that lowering the HLB value increases the log P value of EGCG and results in higher $I_{50}$ values in Henrietta Lacks (HeLa) cancer cells than that of EGCG. Surfactant-modified EGCG-HLB 6 pro $\sigma_{4}$ duced faster and deeper skin penetration than EGCG. Conclusion: Modification of log P value using a combination of Tween 80 and Span 80 improved cytotoxicity and topical delivery of EGCG.
\end{abstract}

First draft submitted: 12 March 2019; Accepted for publication: 5 July 2019; Published online: 19 July 2019

Keywords: (-)-epigallocatechin gallate $\bullet$ cytotoxicity $\bullet$ partition coefficient $\bullet$ penetration $\bullet$ Span $80 \bullet$ Tween 80

Cervical cancer has a high prevalence, constituting the fourth most dangerous form of cancer in women [1]. It has been reported that this form of cancer ranks as the second most common among women in Indonesia, with approximately 18,279 of 32,469 cases proving fatal every year [2]. A recent report confirmed an increase in the number of cervical cancer cases from 702,207 in 2014 to $1,325,776$ in 2015 [3].

Cervical cancer is a malignancy that affects the cervix, the lowest part of uterus which protrudes from the top of the vaginal canal [4] and can develop due to infection caused by human papillomavirus. Different forms of treatment for cervical cancer, namely, surgery, radiotherapy, chemotherapy and chemoradiotherapy, are applicable at successive stages of the disease. However, chemoradiotherapy has been known to affect normal cells causing toxic effects to healthy organs or tissues. The development of natural products for use during chemotherapy is currently progressing rapidly due to the large naturally occurring sources of such products. One of the natural compounds that have been proven through research to be an effective component of chemotherapy is epigallocatechin gallate (EGCG) $[5,6]$.

EGCG represents the largest catechin component of green tea (Camellia sinensis) and is widely known to be a potent antioxidant. EGCG has been reported as inducing anticancer activity through the mechanism of inhibiting tumorigenesis, proliferation and angiogenesis [7]. EGCG triggers an increase in p 53 gene expression in cells experiencing DNA damage [8]; the main function of p53 being to arrest the cell cycle in response to such damage in order that the cell can be repaired before its replication [9]. Moreover, it has been reported that an apoptosis induction occurs in cases of cervical cancer after the administration of EGCG [10,11].

A topical route, such as intravaginal, is proposed as the most appropriate means of drug administration for EGCG in the treatment of cervical cancer. EGCG is highly soluble in water, $40 \mathrm{~g} / 1$ at $20^{\circ} \mathrm{C}$, and demonstrates good stability at $\mathrm{pH} 3.7\left(25^{\circ} \mathrm{C}\right)$ and $\mathrm{pH} 3.9\left(40^{\circ} \mathrm{C}\right)[12]$. However, the $\log \mathrm{P}$ value of EGCG is 1.1 at $\mathrm{pH} 4.0$. It has been reported by Lane et al. that the optimal lipophilicity for topical drug penetration is indicated by the $\log \mathrm{P}$ value within the range of 2-3 [13]. Moreover, it has been reported that EGCG has low cell membrane permeability [14] 


\begin{tabular}{|c|c|c|c|c|}
\hline \multirow[t]{2}{*}{ HLB value } & \multirow[t]{2}{*}{ EGCG (g) } & \multicolumn{3}{|c|}{ Volume (ml) } \\
\hline & & Tween 80 & Span 80 & Phosphate buffer $\mathrm{pH} 5 \pm 0.5$ \\
\hline 8 & 1 & 2 & 4 & ad 20 \\
\hline 6 & 1 & 1 & 5 & ad 20 \\
\hline 4.3 & 1 & - & 6.0 & ad 20 \\
\hline Control & 1 & - & - & ad 20 \\
\hline
\end{tabular}

EGCG: Epigallocatechin gallate; HLB: Hydrophilic-lipophilic balance.

and is strongly attached to skin tissue, thereby reducing diffusion and the rate of skin permeation [15] due to its polar phenolic group content. Therefore, topical delivery of EGCG in 21 ical cancer therapy should be extremely limited $[10,15]$. The use of microemulsion successfully improves topical delivery of EGCG to the deeper skin layer, due to the use of surfactant as emulsifier and oil phase that facilitates EGCG diffusion [16]. Thus, increasing the lipophilicity of EGCG may improve its permeability.

It has been reported that the addition of surfactant to topical drug preparation can enhance the permeability of the drug [17]. Surfactant is an amphiphilic molecule which possesses either hydrophilic head group or lipophilic tail group properties. Surfactants with high hydro 38 lic-lipophilic balance (HLB) values of 8-18 are more soluble in water and form micelles. On the other hand, surfactants with low $\mathrm{r}_{5}$. B values of 3-6 are more soluble in oil and form inverse micelles. Af-idah et al. reported that the HLB value of a combination of Span 80 and Tween 80 exerted a signific $5^{t}$ influence on the physical stability and skin penetration of caffeine microemulsions [18]. In another report, a combination of Span 80 and Tween 80 with an HLB value of 7 increased glutathione lipophilicity from a $\log \mathrm{P}$ value of -1.4 to one of 2.23, thus improving its skin penetration and resulting in a reduction of MMP-1 [19]. Surfactants can also be used as enhancers capable of changing the nature of the lipid bilayer membranes of stratum corneum, thereby increasing percutaneous permeation [20].

In this study, the permeability of EGCG was improved by increasing the lipophilicity of EGCG through a combination of Span 80 and Tween 80 . The modification of EGCG lipophilicity was indicated by a change in the $\log \mathrm{P}$ value as identified by a partition coefficient test. These modified EGCGs were then evaluated for in vitro cytotoxicity in HeLa cells. Moreover, an in vivo skin penetration study was evaluated to quantify 4 e increase in EGCG penetration by using Rhodamine-B-labeled micelles. Reverse micelle formation involving a combination of Tween 80 and Span 80 has considerable potential as a means of modifying EGCG lipophilicity.

\section{Materials \& methods \\ Material: 28}

EGCG of $98 \%$ purity was purchased from Xi'An Rongsheng Biotechnology Co., Ltd (Xi'an, PR China) and Tween 80 was obtained from Sigma Aldrich (MO, USA). Span 80 was a product from Nanhang Industrial Co., Ltd (Hangzhou, China). Rhodamine- $\mathrm{B}$ and $\mathrm{n}$-octanol were secured from Sigma Althich. The buffer components were $\mathrm{NaH}_{2} \mathrm{PO}_{4} \cdot \mathrm{H}_{2} \mathrm{O}$ and $\mathrm{Na}_{2} \mathrm{HPO}_{4} \cdot 12 \mathrm{H}_{2} \mathrm{O}$ (Merck. Inc., Darmstadt, Germany). All other reagents and solvents used in this study were of the finest available quality.

\section{Prepar 3 on of EGCG reverse micelles}

EGCG was dissolved in buffer phosphate $\mathrm{pH} 5 \pm 0.5$ with a mixture of Tween 80 and Span 80 prepared at different weight ratios, as shown in Table 1 , to form systems with HLB values of 8,6 and 4.3. The HLB system was calculated using the following equation:

$$
\begin{gathered}
\% \text { Tween } 80=\frac{100\left(X-H L B_{\text {span } 80}\right)}{H L B_{\text {Tween } 80}-H L B_{\text {Span } 80}} \\
\% \text { Span } 80=100-\% \text { Tween } 80
\end{gathered}
$$

10

$\mathrm{X}$ refers to the intended HLB value, in other words, 8,6 and 4.3. The HLB values of Span 80 and Tween 80 are 4.3 and 15 , respectively [21]. 
Determination of equilibrium time for apparent partition coefficient study of EGCG

Approximately $10.0 \mathrm{mg}$ of EGCG were dissolved in $10 \mathrm{ml}$ phosphate buffer $\mathrm{pH} 5$, which had previously been saturated with n-octanol to obtain a $1000 \mathrm{ppm}$ EGCG solution. This was then diluted to produce $40 \mathrm{ppm}$ EGCG solution which was added to n-octanol-saturated buffer at a ratio of 1:10 and mixed in a $50 \mathrm{ml}$ Erlenmeyer flask. The mixture was agitated at a frequency of 150 times per minute using a thermoshaker at $37 \pm 175^{\circ} \mathrm{C}$. The buffer solution was subsequently separated from n-octanol after 5, 10, 15, 30, 45 and $60 \mathrm{~min}$. The samples were

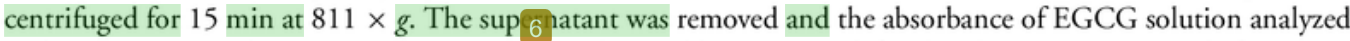
with an UV spectrophotometer at $\lambda=271.5 \mathrm{~nm}$. The equilibrium time, defined as the minimum period of agitation required to achieve no significant difference of EGCG levels during the study, was then determined.

\section{Det 3 nination of apparent partition coefficient of EGCG after surfactant addition}

The determination of apparent partition coefficient was carried out in phosphate buffer $\mathrm{pH} 5.0 \pm 0.05$ saturated with $\mathrm{n}$-octanol as the water phase, using a shake flask method $[19,22]$. The initial concentrations of EGCG were 1000, 2000 and 10,000 ppm for HLB 8, 6 and 4.3, respectively. The EGCG sol 3 ion was put into a glass vial and added to $1.0 \mathrm{ml}$ of n-octanol as the organic phase, which was saturated with phosphate buffer solution $\mathrm{pH}$ $5.0 \pm 0.5$. The mixture was then agitated with a thermoshaker at 150 r.p.m. at $37 \pm 0.5^{\circ} \mathrm{C}$ for $30 \mathrm{~min}$. The water phase was separated from the organic phase by centrifuging samples for $15 \mathrm{~min}$ at $811 \times \mathrm{g}$. The EGCG level in the water phase was then quantified using a UV spectrophotometer at $\lambda=271.5 \mathrm{~nm}$.

The $\log$ P value was calculated based on the following formula [19]:

$$
\log P=\log \frac{\left(C_{w}^{o}-C_{w}^{1}\right) \cdot V_{w}}{C_{w}^{1} \cdot V_{o}}
$$

where $\mathrm{P}$ is the apparent partition coefficient; $C_{w}^{o}$ is the drug con 14 tration in water phase before equilibrium; $C_{w}^{1}$ is the drug concentration in water phase after equilibrium; $V_{w}$ is the volume of phosphate buffer as the water phase; and $V_{0}$ is the volume of n-octanol as the organic phase.

In vitro cytotoxicity study on HeLa cells

HeLa cells were obtained fror 20 aboratory for Joint Basic Experiments, Faculty of Pharmacy, Universitas 8 irlangga (Surabaya, Indonesia). HeLa cells were cultured in the Rosewell Park Memorial Institute 1640 medium with $10 \%$ heat-inactiva $1 \mathrm{detal}$ bovine serum, penicillin $(100 \mathrm{units} / \mathrm{ml})$, streptomycin $(100 \mu \mathrm{g} / \mathrm{ml})$ and amphoteric 1 B $(2.5 \mu \mathrm{g} / \mathrm{ml})$ in a humidified atmosphere containing $5 \% \mathrm{CO}_{2}$ at $37^{\circ} \mathrm{C}$. For the in vitro cytotoxic assay, HeLa cells were seeded separately at a density of $5 \times 10^{3}$ cells per well in 96-well plates and maintained in the medium for 24 h before treatment.

To examine the cytotoxicity, the cells were treated with medium containing EGCG at concentrations of 10, 20, 40, 80 and 100 ppm in EGCG, surfactant-modified EGCG-HLB 4.3, or surfactant-modifie 1 EGCG-HLB 6. To aid sample solubility in medium, surfactant-modified EGCG wa 15 dded to DMSO. The cells were then incubated for $48 \mathrm{~h}$. After treatment, the cell number was determined using 3-(4,5-dime 6 lthiazol-2-yl)-2,5-diphenyltetrazolium bromide assay. On 9 onclusion of the culture media incubation period, the medium was discarded and replaced with $100 \mu \mathrm{l}$ of 5\% 3-(4,5-dimethylthiazol-2-yl)-2,5- (44)enyltetrazolium bromide solution (Sigma Aldrich) in each well. Thereafter, the plate was plac 12 in an incubator for $4 \mathrm{~h}$, until the formation of formazan crystals was observed under a light microscope. Then, a $100 \mu \mathrm{l}$ of $10 \%$ sodium dodecyl sulfate solution in $0.01 \mathrm{M} \mathrm{H}_{37}$ was subsequently being added to each well. The plate was wrapped in aluminum foil, incubafl overnight in the dark at room temperature and its absorbance measured with an ELISA reader at $\mathrm{l}=595 \mathrm{~nm}$. Cell viability was expressed relative to the absorbance of untreated cells and media, while the concentration leading to $50 \%$ cell viability (IC ( $_{0}$ ) was also calculated.

40

\section{In vivo skin penetration study}

For the purposes of the in vivo skin penetration study, Rhodamine-B-labeled micelle was prepared to enable evaluation of the ability of surfactant-modified EGCG to permeate the skin of rats. Rhodamine-B was initially dissolved with $10 \mathrm{ml}$ phosphate buffer $\mathrm{pH} 5$ to produce a $0.01 \%$ Rhodamine-B solution. Approximately $1 \mathrm{~g}$ of EGCG was then added and the mixture stirre 6 intil homogenous with roughly $1 \mathrm{ml}$ of Tween 80 and $5 \mathrm{ml}$ of Span 80 being introduced. Phosphate buffer $\mathrm{pH} 5$ was added to produce a final mixture with a volume of $20 \mathrm{ml}$ which 


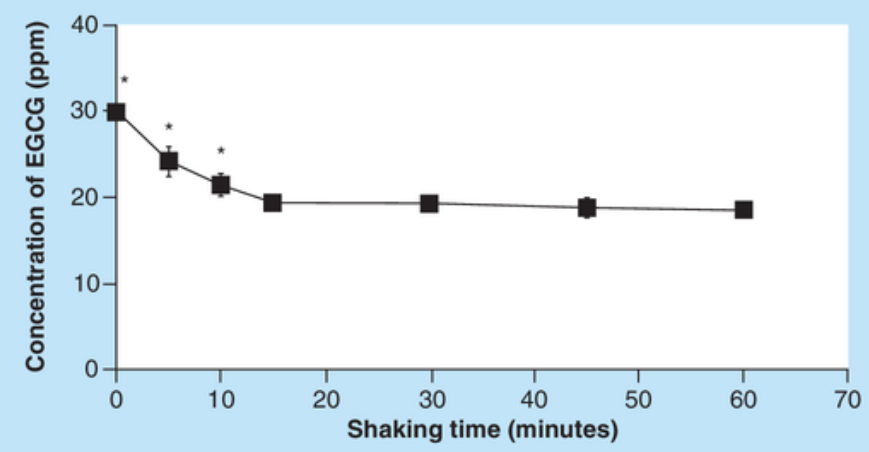

Figure 1. The 3 tion between shaking time (minutes) and epigallocatechin gallate concentration ( $\mathrm{ppm}$ ) observed in a mixture of n-octanol and $0.01 \mathrm{M}$ phosphate buffer $\mathrm{pH} 5 \pm 0.05$ at temperature of $37 \pm 0.5^{\circ} \mathrm{C}$. All data were in three replicates.

${ }^{*} \mathrm{p}<0.05$ versus $60 \mathrm{~min}$.

\section{6}

was then stirred at 1000 r.p.m. for 15 min. The mixtures of Rhodamine-B and surfactant and Rhodamine-B, which had been freeze-dried to produce a sample that had semi-solid consistency, and EGCG were used as the control groups.

The research subjects were Wistar rats (male: 8-10 weeks old and 150-200 g in weight) purchased from the Animal Laboratory, Faculty of Pharmacy, $\mathrm{U}_{2}$ versitas Airlangga (Surabaya, Indonesia). All subjects were maintained and treated in conditions which complied with the guiding principles for the care and use of laboratory animals as established by Faculty of Veterinary Science, Universitas Airlangga, Indonesia.

The subjects were divided into three groups ea ${ }_{26}$ ontaining four subjects, representing surfactant, EGCG, and surfactant-modified EGCG HLB 6 treatment for Group 1, Group 2 and Group 3, respectively. The samples were applied non-occlusively to a $6 \mathrm{~cm}^{2}$ area of the abdominal skin of subjects. To avoid sample spilled out from the designated area, the rat's abdomen was covered with a water-impermeable plastic paper that had a hole created at an exact siz $132 \times 3 \mathrm{~cm}^{2}$ for sample application. The dose was equal to $50 \mathrm{mg}$ of EGCG per $\mathrm{kg}$. During the study, all subjects were anesthetized wi $18 \mathrm{n}$ intramuscular injection of Ketamine $(20 \mathrm{mg} / \mathrm{kg}$ body weight). After $2 \mathrm{~h}$ of drug administrati11, the subjects were sacrificed by means of cervical dislocation. The section of skin tissue was then excised and embedded in an optimal cutting temperature (OCT) compound (Tissue Tek ${ }^{\circledR}$, Sakura Finetechnical Co., Tokyo, Japan). It was then processed by frozen sectioning of $5 \mu \mathrm{m}$ thickness. The prepared skin tissue slices were then observed under a fluorescence microscope (FSX100, Olympus, Tokyo, Japan).

43

\section{Statistical analysis}

All data were in the form of 3-4 replicates and presented as the $\mathrm{m}_{25} \pm \mathrm{SD}$. To evaluate the significance of the difference, the da 42 vere analyzed by one way ANOVA followed by least significant difference test with $\mathrm{p}$-value $<$ 0.05 and $<0.01$ using SPSS Software v.21.0.

\section{Results \& discussion}

7 termination of equilibrium time \& apparent partition coefficient of EGCG

To increase the skin penetration of EGCG for topical drug delivery, the modification of EGCG hydrophobicity was investigated by reverse micelle method. First, the equilibrium time required for evaluating apparent partition coefficient or $\log$ P of EGCG and surfactant-modified EGCG was determined. It has been reported that EGCG is stable in low $\mathrm{pH}$ condition, in other words, $\mathrm{pH} 2-5.5$ and it is unstable in alkaline condition [24]. In addition, for the a 24 ptability of topical vaginal product, the $\mathrm{pH}$ should fall within 4-6 [23]. Therefore, in this study, we prepared EGCG in phosphate buffer $\mathrm{pH}$ 5. The equilibrium time of EGCG in a phosphate buffer $\mathrm{pH} 5 / \mathrm{n}$-octanol at $37 \pm 0.5^{\circ} \mathrm{C}$ was reached after constant EGCG levels had been obtained. As shown in Figure 1, the EGCG concentrations at 15,30, 35 and 60 min were not significantly different. Consequently, it can be postulated that 


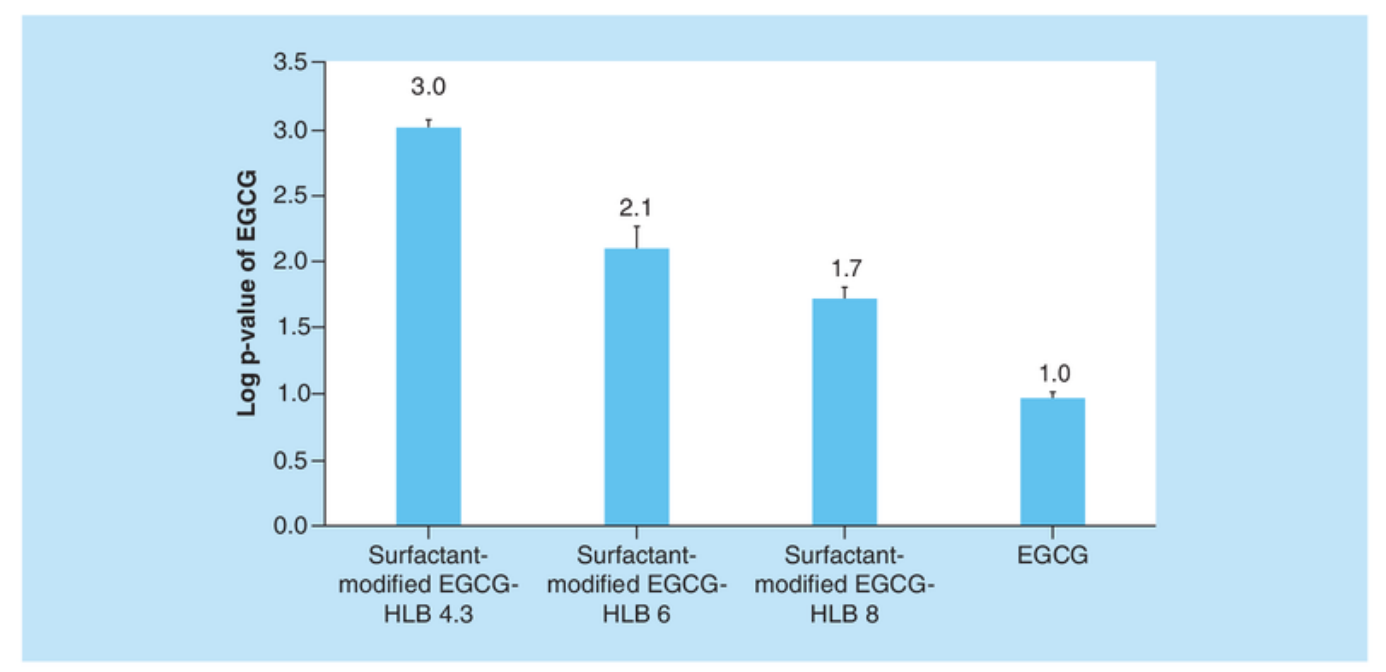

Figure 2. The log $\mathrm{P}$ values of epigallocatechin gallate and surfactant-modified epigallocatechi 3 gallate-hydrophilic-lipophilic balance $4.3,6$ and 8 . The study was performed by using a mixture of n-octanol and $0.01 \mathrm{M}$ phosphate buffer $\mathrm{pH} 5 \pm 0.05$ at $37 \pm 0.5^{\circ} \mathrm{C}$ and agitating in a thermoshaker for $30 \mathrm{~min}$. All data were in three replicates.

after 15 min the EGCG levels were constant. During this study, $30 \mathrm{~min}$ was chosen as the equilibrium time for further experiments.

\section{Determination of the EGCG partition coefficient at pH $5.0 \pm 0.5$}

HLB represents the proportion of hydrophilic and lipophilic nature of surfactants [21]. On the other hand, cancer cells, in addition to other eukaryote cells, possess a membrane consisting of lipoprotein that requires a drug to exhibit similar lipophilicity in permeating it [25]. Thus, preparing drug ${ }_{5}$ urfactant mixture of different HLB values may modify the hydrophobicity of EGCG. The success with which the combination of Span 80 and Tween 80 altered the apparent partition coefficient of EGCG in phosphate buffer $\mathrm{pH} 5 / \mathrm{n}$-octanol media was investigated. Determ 23 tion of the apparent partition coefficient $\left(\log \mathrm{P}_{\text {app }}\right.$ ) was conduct by agitating samples equal to $40 \mathrm{ppm}$ EGCG in $0.01 \mathrm{M}$ phosphate buffer solution $\mathrm{pH} 5.0$ as the water phase and n-octanol as the oil phase at $37 \pm 0.5^{\circ} \mathrm{C}$ for $30 \mathrm{~min}$. It can be seen in Figure 2, that an increasing HLB value redu 35 the $\log$ P value of EGCG. The $\log$ P value obtained was subsequently used to determine the selected formula for the study of in vivo skin penetration. From the results, it can be proposed that modifying EGCG in the HLB values of 4.3 and 6 produced $\log$ P values between 2 and 3, that ar 34 milar to the $\log$ P value of skin. This may be due to the entrapment of EGCG, that is highly soluble in water, in the inner core of the reverse micelle which probably of the water in oil (w/o) micellar type. This condition creates 'like dissolve like' phenomena between EGCG and more lipophilic components, in other words, n-octanol, in the oil phase [26]. It has been known that Span 80 and Tween 80, which act as the surfactants, contain hydrophilic and lipophilic groups [27] that may facilitate the interaction between EGCG and $\mathrm{n}$-octanol. Therefore, it renders the nature properties of EGCG more hydrophobic.

The cytotoxicity of EGCG on HeLa cells

It has been reported that most EGCG cellular activity is related to its very high concentration of hundreds of micromoles [6,14], which indicates that its polar phenolic hydroxyl group limits EGCG permeability across cell membrane. Improving the hydrophobicity of the system by, for example, using liposom as fusogenic lipid carrier, improved the membrane permeability of EGCG, thus producing effective cellular activity of EGCG at low concentrations [14].

During this study, the log P value was modified by using surfactant combinations of Span 80-Tween 80 prepared at different HLB values. This constituted an effort to increase the lipophilicity of EGCG [28], which was evaluated by phosphate buffer $\mathrm{pH} 5 / \mathrm{n}$-octanol partition study, in order to improve the membrane permeability and cytotoxicity 


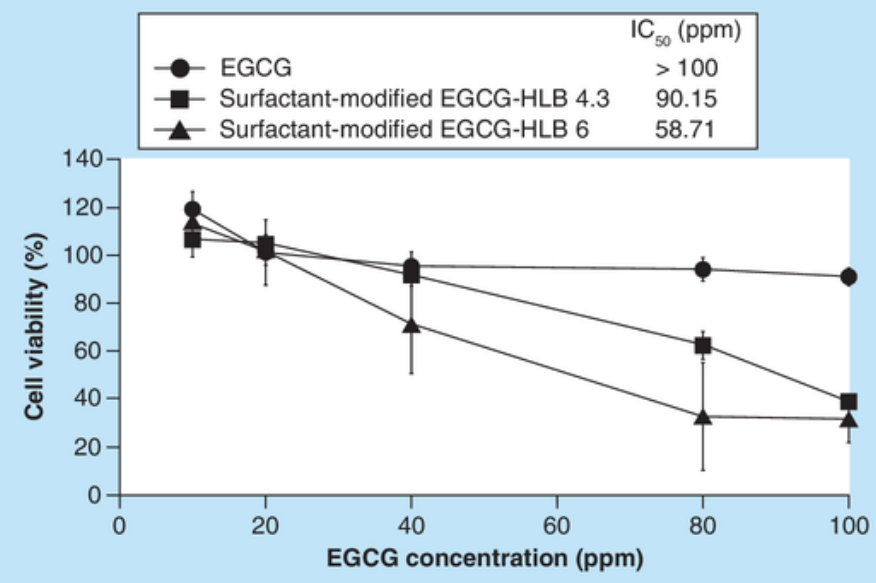

Figure 3. In vitro cytotoxicities of epigallocatechin gallate, surfactant-modified epigallocatechin gallate-hydrophilic-lipophilic ba 33 e 4.3 and surfactant-modified epigallocatechin gallate-hydrophilic-lipophilic balance 6 in HeLa cells. Samples were added to the cells at concentrations of epigallocatechin gallate (EGCG) between 10 and $100 \mathrm{ppm}$ and then incubated for $48 \mathrm{~h}$. Each value represents a mean \pm SD of four replicates for surfactant-modified EGCG-HLB 4.3 and surfactant-modified EGCG-HLB 6 and three replicates for EGCG. HLB: Hydrophilic-lipophilic balance.

of EGCG. The results showed that surfactant-modified EGCG can produce higher cytotoxicity than native EGCG. The addition of Span 80 and Tween 80 increased the cytotoxicity of EGCG as shown in Figure 3. Surfactantmodified EGCG produced high cytotoxic effects with the $\mathrm{IC}_{50}$ values of 90.15 and $58.71 \mathrm{ppm}$ for HLB value of 4.3 and 6, respectively. On the other hand, EGCG demonstrated low cytotoxicity, as indicated by its IC $_{50}$ value $>100 \mathrm{ppm}$. This study also evaluated the cell viability of control groups, which are DMSO and surfactants, and there were no remarkable cytotoxic effects on HeLa cells (data not shown). This is possibly due to the alteration of EGCG hydrophobicity, which became more lipophilic than native EGCG, thus increasing its ability to penetrate cell membrane. Koga et al. have reported that at nonsolubilizing concentrations Span and Tween altered membrane lipid fluidity related to hydrophobicity as refle 41 d by the HLB number of these surfactants [29]. The lipid membrane changes were predominantly located in the outer leaflet of lipid membrane due to the interaction between fatty acid groups of surfactants and polar phospholipid head group of cell membrane. In addition, modifying the HLB system at values of 4.3 and 6 produced almost half of $\mathrm{IC}_{50}$ although their respective relatively close $\log \mathrm{P}$ values were 3 and 2.1. A previous report stated that a $\log \mathrm{P}$ value of 2.5 becomes an intersection where, below this point, the penetrants will be favorably distributed within the dermis, while above it substances will more probably be partitioned into the epidermis [30], which is more lipophilic than a dermis stratum. The cell membrane is likely to possess similar characteristics to the dermis area, rather tha 32 e epidermis, due to its water and lipoprotein content.

It has been previously reported that cancerous cell are more sensitive to oxidative stress than normal cells and EGCG produce extracellular and intracellular oxidative stress, either through the production of hydrogen peroxide or semiquinone radicals [6]. A repo 22 lso exists which argued that an increase in oxidative stress production occurs in cancer cell induced by EGCG while reducing reactive oxygen species levels in normal cells [31]. However, the cytotoxicity of surfactant-modified EGCG in normal cells was undetermined in this study. Further investigation into oxidative degradation stability of surfactant-modified EGCG and its cell cytotoxic sensitivity are required. The formation of reverse micelle improved cell permeability and entrapping EGCG inside may offer some protection for EGCG from oxidative degradation.

\section{Penetration of EGCG into rat skin}

It has been determined that $\log \mathrm{P}$ indicates the lipophilicity of penetrants that plays a major role in skin permeation. Skin permeation of EGCG has been reported to be very low due to a high bound percentage (approaching 90\%) of EGCG in skin tissue, thus reducing the degree and rate of skin permeation of EGCG during the diffusion 
Figure 4. The photomicrographs of Rhodamine-labeled surfactant, surfactant-modified epigallocatechin gallate at an hydrophilic-lipophilic balance value of 6 and epigallocatechin gallate observed $2 \mathrm{~h}$ after topical application of samples into Wistar rat abdome 31 uring an in vivo skin penetration study. The samples were observed through a fluorescence microscope at a magnification of $42 \times$. The Rhodamine-labeled EGCG is indicated by a white arrow.

EGCG: Epigallocatechin gallate.

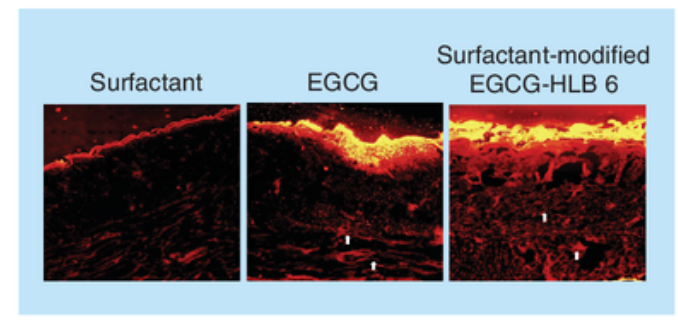

process [15]. In this study, increasing the hydrophobicity of EGCG through modification of the $\log P$ value improved its penetration of the rat's skin. The skin penetration of EGCG and its modified forms were evaluated by observing the EGCG fluorescence of the skin tissue preparation after $2 \mathrm{~h}$ of application. This constitutes a prestudy to evaluate the permeation rate of surfactant-modified EGCG for topical vaginal routes. As shown in Figure 4, there was an increase in skin penetration for modified EGCG, which had been processed with surfactant addition, $2 \mathrm{~h}$ after application. We have also evaluated for skin penetration of these samples $5 \mathrm{~h}$ after application, and it resulted in no significant different from those of $2 \mathrm{~h}$ (data not shown). This modified EGCG demonstrated more intense fluorescence than EGCG solution. It was confirmed that surfactant addition did not affect observation of the fluorescence of the samples.

It is known that skin tissue consists of lipoprotein that has amphiphilic properties which probably cause the EGCG trapped in the reverse micelle core to more easily penetrate the skin layer than the native EGCG. In addition to this mechanism, the surfactants can act as enhancers that affect the integrity of the stratum corneum. It has been reported that the presence of surfactant, which has the function of an enhancer, also plays a role in the EGCG penetration process [20]. Therefore, it also improves the skin penetration of EGCG.

\section{Conclusion}

In sur 4 hary, changes in the partition coefficient for improving the permeability of EGCG by micelle formation using a combination of Twe 1680 and Span 80 were analyzed. The reverse micelle method successfully increased the lipophilicity of EGCG. The use of surfactant combination with a HLB value of 6 resulted in the $\log$ P value of EGCG being closest to that of skin, at 2-3, and producing higher cytotoxicity and more rapid and deeper skin penetration compared with native EGCG. Further evaluation of the anticancer activity of this modified EGCG is required to produce a high level of success of cervical cancer therapy.

\section{Future perspective}

Developing the topical delivery of EGCG by means of creams or in ovule-like dosage form represents an effective alternative means of supporting the treatment of cervical cancer. This study produced a positive approach to effectively increasing the EGCG permeability through skin by altering the $\log$ P value using a surfactant combination of Tween 830 nd Span 80 . As a preliminary investigation, this research represents a stepping stone in applying science to the development of therapeutic products for the treatment of cancer. Further research is definitely required to perfect and comprehensively define the delivery system. Evaluating drug tissue distribution would enable quantification of the effectiveness of the system in improving the skin penetration of EGCG. Moreover, further investigation of the 29 rfactant-drug assembles that may affect the morphological structures of EGCGsurfactant mixtures would play an important role in determining the detailed interaction between EGCG and surfactants, and the manner in which the system could improve the skin penetration of EGCG.

Financial \& competing interests disclosure

This work was supported by Universitas Airlangga, Indonesia (grant number 86/UN3/2018, 2018). The authors have no other relevant affiliations or financial involvement with any organization or entity with a financial interest in or financial conflict with the subject matter or materials discussed in the manuscript apart from those disclosed.

No writing assistance was utilized in the production of this manuscript. 
Ethical conduct of research

The animal experimental investigation used in this study was reviewed and complied with the guiding principles for the care and use of laboratory animals as set up by the formal ethics review committee on animal research of the Faculty of Veterinary Science, Universitas Airlangga, Indonesia.

\section{Summary points}

Topical delivery of epigallo 39 chin gallate for cervical cancer

- Epigallocatechin gallate (EGCG) has been reported to produce an apoptosis induction in cervical cancer.

- EGCG has high water solubility but has low permeability limiting topical delivery for therapeutic treatment of cervical cancer.

Log P value determining drug lipophilicity \& skin penetration

- The optimal lipophilicity for topical drug penetration is indicated by the log P value within the range of 2-3.

- It has been reported that the use of surfactant could modify the log P value that influences physical stability, 4 lipophilicity and skin penetration of a drug.

Combination of Tween 80 \& Span 80 for improving permeabi 4 i/ of EGCG

- Lowering the hydrophilic-lipophilic balance value by using a combination of Tween 80 and Span 80 increased the log P value of EGCG.

- Increasing the log P value of EGCG by addition of Tween 80 and Span 80 enhanced the cytotoxicity of EGCG.

- EGCG modified with Tween 80 and Span 80 produced improved penetration of a rat's skin.

\section{References}

Papers of special note have been highlighted as: $\bullet$ of interest; $\bullet \bullet$ of considerable interest

1. Cervical cancer. https://www.who.int/cancer/prevention/diagnosis-screening/cervical-cancer/en/

2. Indonesia-human papillomavirus and related cancers, Fact Sheet 2018. https://hpvcentre.net/statistics/reports/IDN_FS.pdf

3. Wahidin M. Cervical cancer screening financing in Indonesia. J. Glob. Oncol. 4(2), 93s-93s (2018),

4. Casey PM, Long ME, Marnach ML. Concise review for clinicians-abnormal cervical appearance: what to do, when to worry? Mayo Clin. Proc. 86(2), 147-151 (2011).

5. McDonnell AM, Pyles HM, Diaz-cruz ES, Barton CE. Synergistically to inhibit the growth of cervical cancer cells in culture. Molecules 24(1580), 1-7 (2019).

6. Weisburg JH, Weissman DB, Sedaghat T, Babich $\mathrm{H}$. In vitro cytotoxicity of epigallocatechin gallate and tea extracts to cancerous and normal cells from the human oral cavity. Basic Clin. Pharmacol. Toxicol. 95(4), 191-200 (2004).

- Provides information regarding selective anticancer activitiy of epigallocatechin gallate (EGCG) to malignant human oral cavity cells through prooxidant mechanism.

7. Wang Y, Lu J, Liang Y. Suppressive effects of EGCG on cervical cancer. Molecules 23(2334), 1-17 (2018).

8. Thakur VS, Gupta K, Gupta S. Green tea polyphenols increase p53 transcriptional activity and acetylation by suppressing class I histone deacetylases. Int. J. Oncol. 41(1), 353-361 (2012).

9. Williams AB, Schumacher B. p53 in the DNA-damage-repair process. Cold Spring Harb. Perspect. Med. 6(5), 1-32 (2016).

10. Ahn W-S, Yoo J, Huh S-W et al. Protective effects of green tea extracts (polyphenon E and EGCG) on human cervical lesions. Eur. J. Cancer Prev. 12(5), 383-390 (2019).

11. Asif Siddiqui F, Naim M, Islam N. Apoptotic effect of green tea polyphenol (EGCG) on cervical carcinoma cells. Diagn. Cytopathol. 39(7), 482-488 (2011).

12. Zeng L, Ma M, Li C, Luo L. Stability of tea polyphenols solution with different $\mathrm{pH}$ at different temperatures. Int. J. Food Prop. 20(1), 1-18 (2016).

- Provides information regarding the stability of EGCG in low acid $\mathrm{pH}$ condition.

13. Lane ME, Santos P, Watkinson AC, Hadgraft J. Passive skin permeation enhancement. In: Topical and Transdermal Drug Delivery. Benson HA, Watkinson AC (Eds). John Wiley \& Sons, Inc. NJ, USA (2012).

14. Li X, Tang S, Wang Q et al. Identification of eEpigallocatechin-3-gallate as an inhibitor of phosphoglycerate mutase 1. Front. Pharmacol. 8, 1-9 (2017).

- Provides information regarding low permeability of EGCG to across cell membrane.

15. Yoshino S, Mitoma T, Tsuruta K, Todo H, Sugibayashi K. Effect of emulsification on the skin permeation and UV protection of catechin. Pharm. Dev. Technol. 19(4), 395-400 (2014).

- Provides important information about permeation of EGCG in rat's skin, in which high fraction of its amount are bound to skin tissue, and the use of oil-in-water emulsion successfully improves skin penetration EGCG. 
16. Scalia S, Trotta V, Bianchi A. In vivo human skin penetration of (-)-epigallocatechin-3-gallate from topical formulations. Acta Pharm. 64, 257-265 (2014).

- Provides comparison data of human skin permeation of EGCG between gel and oil-in-water cream formulation that states oil-in-water cream enhances EGCG penetration into deeper layer of stratum corneum.

17. Ghosh PK, Murthy RS. Microemulsions: a potential drug delivery system. Curr. Drug Deliv. 3(2), 167-180 (2006).

18. Af-Idah BM, Nurahmanto D, Ameliana L. Optimasi nilai HLB kombinasi surfaktan dan konsentrasi isopropil miristat pada mikroemulsi kafein. Faculty of Pharmacy Bachelor. 1-69 (2016).

19. Nugrahaeni F, Hariyadi DM, Rosita N. Partition coefficient and glutathione penetration of topical antiaging: preformulation study. Int. J. Drug Deliv. Technol. 8, 39-43 (2018).

- Provides early data about hydrophilic-lipophilic balance modification to improve lipophilicy of glutathione, which has log $\mathbf{P}$ value of 1.22 changed to 2.26 , and this modification successfully improves skin penetration of glutathione and its activity expressed by decreased expression of matrix metalloproteinase-1 of UV-radiated mice skin.

20. Lane ME. Skin penetration enhancers. Int. J. Pharm. 447(1), 12-21 (2013).

21. Nollet M, Boulghobra H, Calligaro E, Rodier J. An efficient method to determine the hydrophile-lipophile balance of surfactants using the phase inversion temperature deviation of CiEj/n-octane /water emulsions. Int. J. Cosmet. Sci. 41(2), 99-18 (2019).

22. Andrés A, Rosés M, Ràfols $\mathrm{C}$ et al. Setup and validation of shake-flask procedures for the determination of partition coefficients (log D) from low drug amounts. Eur. J. Pharm. Sci. 76, 181-191 (2015).

23. Co JT, Bugayong JCC, Monge GC. Acceptability and effectivity of four feminine wash products in maintaining the physiologic vaginal $\mathrm{pH}$ of reproductive and menopausal age women. Acta Medica Philippina 52(6), 533-540 (2018).

24. Krupkovaa O, Fergusona SJ, Wuertz-Kozaka K. Stability of (-)-epigallocatechin gallate and its activity in liquid formulations and delivery systems. J. Nutr. Biochem. 37, 1-12 (2016).

25. Rivel T, Ramseyer C, Yesylevskyy S. The asymmetry of plasma membranes and their cholesterol content infuence the uptake of cisplatin. Sci. Rep. 9(5627), 1-14 (2019).

26. Valsaraj KT, Thibodeaux LJ. Relationship between micelle-water and octanol-water partition constants for hydrophobic organics of environmental interest. Wat. Res. 23(2), 183-189 (1989).

27. Mahdi ES, Sakeena MHF, Abdulkarim MF, Abdullah GZ, Sattar MA, Noor AM. Effect of surfactant and surfactant blends on pseudoternary phase diagram behavior of newly synthesized palm kernel oil esters. Drug Des. Devel. Ther. 5, 311-323 (2011).

28. Di L, Kerns EH. Lipophilicity methods. In: Drug-Like Properties Concepts, Structure Design and Methods from ADME to Toxicity Optimization. Di L, Kerns EH (Eds). Academic Press California, CA, USA (2016).

29. Koga K, Murakami M. Contribution of hydrophobicity of nonionic detergents to membrane lipid fluidity and disopyramide uptake by rat intestinal brush-border membrane. Biol. Pharm. Bull. 20(6), 674-679 (1997).

-. Provides information regarding the mechanism of surfactant for improving drug permeability, which at nonsolubilizing concentration, surfactant changes the fluidity of the outer layer of lipophilic leaflet of cell membrane, and it states that hydrophobicity, represented by hydrophilic-lipophilic balance number, becomes the determinant factor.

30. Cross SE, Magnusson BM, Winckle G, Anissimov Y, Roberts MS. Determination of the effect of lipophilicity on the in vitro permeability and tissue reservoir characteristics of topically applied solutes in human skin layers. J. Invest. Dermatol. 120(5), 759-764 (2003).

31. Yamamoto T, Hsu S, Lewis J et al. Green tea polyphenol causes differential oxidative environments in tumor versus normal epithelial cells. J. Pharmacol. Exp. Ther. 307(1), 230-236 (2003).

- Provides information regarding oxidative stress induced by EGCG and EGCG can act as radical oxygen species inducer of suppressor depending on cell types, in other words, cancerous or normal cells. 
Enhancing skin penetration of epigallocatechin gallate by modifying partition coefficient using reverse micelle method

ORIGINALITY REPORT

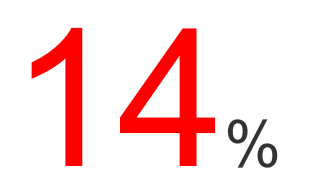

SIMILARITY INDEX
$9 \%$

INTERNET SOURCES
$10 \%$

PUBLICATIONS
$2 \%$

STUDENT PAPERS

PRIMARY SOURCES

1 file.scirp.org

Internet Source

2 www.avensonline.org

Internet Source

3 Submitted to Universitas Airlangga Student Paper

Yadav Vivek Ramshankar, Sarasija Suresh,

Kshama Devi. "Novel Self-emulsifying

Formulation of Curcumin with Improved

Dissolution, Antiangiogenic and Antiinflammatory Activity", Clinical Research and

Regulatory Affairs, 2008

Publication

5 Esmaeili, Hossein, Feridun Esmaeilzadeh, and

Dariush Mowla. "Effect of Surfactant on Stability and Size Distribution of Gas Condensate

Droplets in Water", Journal of Chemical \&

Engineering Data, 2014. 
7 Percutaneous Penetration Enhancers Chemical Methods in Penetration Enhancement, 2015.

8 molpharm.aspetjournals.org

9 citeseerx.ist.psu.edu

10 Jain, Subheet, Parijat Jain, R. B.

Umamaheshwari, and N. K. Jain.

"Transfersomes-A Novel Vesicular Carrier for

Enhanced Transdermal Delivery: Development,

Characterization, and Performance Evaluation",

Drug Development and Industrial Pharmacy,

2003.

Publication

11 www.ncbi.nlm.nih.gov

Internet Source

12 pinnacle.allenpress.com

Internet Source

13 aac.asm.org

Internet Source

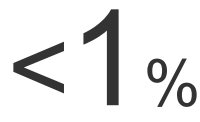

D. Attwood, A. T. Florence. "Surfactant

Systems", Springer Nature, 1983 
15 mdpi.com

16 Mona Qushawy, Ali Nasr, Mohammed Abd-

Alhaseeb, Shady Swidan. "Design, Optimization

and Characterization of a Transfersomal Gel

Using Miconazole Nitrate for the Treatment of

Candida Skin Infections", Pharmaceutics, 2018

Publication

17

Ren, Qiuyue, Chunli Deng, Lin Meng, Yanzuo

Chen, Liangcen Chen, Xianyi Sha, and Xiaoling

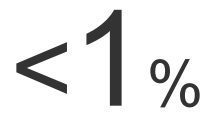

Fang. "In Vitro, Ex Vivo, and In Vivo Evaluation

of the Effect of Saturated Fat Acid Chain Length

on the Transdermal Behavior of Ibuprofen-

Loaded Microemulsions", Journal of

Pharmaceutical Sciences, 2014.

Publication

18 ijbpas.com

Internet Source

19 journals.ekb.eg

Internet Source

20 www.hudba-jistebnik.cz

21 Puri, Ashana, Hiep X. Nguyen, and Ajay K.

Banga. "Microneedle-mediated intradermal delivery of epigallocatechin-3-gallate", 
International Journal of Cosmetic Science, 2016.

Publication

22 dermage.com.br

Internet Source

$<1 \%$

23 real-j.mtak.hu

Internet Source

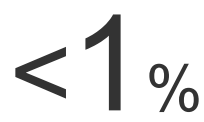

24 Judith Delius, Oliver Frank, Thomas Hofmann.

"Label-free quantitative 1H NMR spectroscopy to study low-affinity ligand-protein interactions in

solution: A contribution to the mechanism of

polyphenol-mediated astringency", PLOS ONE,

2017

Publication

25 patents.google.com

Internet Source

26 www.freepatentsonline.com

Internet Source

27 Steve Huysman, Francis Vanryckeghem, Ellen

De Paepe, Foppe Smedes et al. "Hydrophilic

Divinylbenzene for Equilibrium Sorption of

Emerging Organic Contaminants in Aquatic

Matrices", Environmental Science \&

Technology, 2019

Publication

Jiajun Zeng, Huanhua Xu, Yu Cai, Yan Xuan, Jia Liu, Yue Gao, Qingxian Luan. "The Effect of 
Ultrasound, Oxygen and Sunlight on the Stability of (-)-Epigallocatechin Gallate", Molecules, 2018

Publication

29 edepot.wur.nl

Internet Source

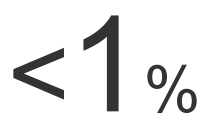

30 effiloop.com

Internet Source

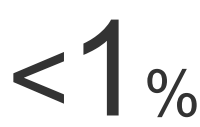

31 www.dovepress.com

Internet Source

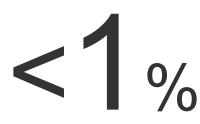

32 icrpstudy.com

Internet Source

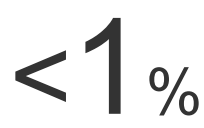

33 www.research-collection.ethz.ch

Internet Source

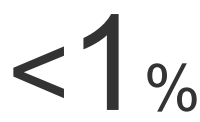

34 ecommons.usask.ca

Internet Source

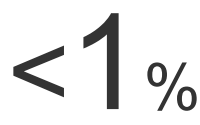

35 www2.mst.dk

Internet Source

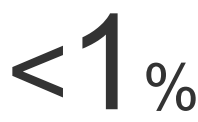

36 M. Nollet, H. Boulghobra, E. Calligaro, J.-D.

Rodier. " An efficient method to determine the

Hydrophile-Lipophile Balance of surfactants

using the phase inversion temperature deviation

of C E / -octane/water emulsions ", International

Journal of Cosmetic Science, 2019

Publication 
38 Flaurance Kenfack, Siegfried Vieth. "Synthesis of eucryptite spheres", Journal of Materials Science, 2008

Publication

39

Sailendra N. Nichenametla, Todd G. Taruscio, Danny L. Barney, Jerry H. Exon. "A Review of the Effects and Mechanisms of Polyphenolics in Cancer", Critical Reviews in Food Science and Nutrition, 2006

Publication

T. Hatahet, M. Morille, A. Hommoss, J.M.

Devoisselle, R.H. Müller, S. Bégu. "Liposomes, lipid nanocapsules and smartCrystals $\AA$ : A comparative study for an effective quercetin delivery to the skin", International Journal of Pharmaceutics, 2018 Publication

Da-Wei Zhang, Kenichi Nunoya, Monika Vasa, Hong-Mei Gu, Susan P. C. Cole, Roger G. Deeley. "MUTATIONAL ANALYSIS OF POLAR AMINO ACID RESIDUES WITHIN PREDICTED TRANSMEMBRANE HELICES 10 AND 16 OF MULTIDRUG RESISTANCE PROTEIN 1 (ABCC1): EFFECT ON SUBSTRATE SPECIFICITY", Drug Metabolism and 
Disposition, 2006

42

"Evaluation of Transfersome and

Protransfersome for Percutaneous Delivery

ofCisplatin in Hairless Mice ", Journal of

Pharmaceutics \& Pharmacology, 2015

Publication

43

Miatmoko, Andang, Kumi Kawano, Etsuo

Yonemochi, and Yoshiyuki Hattori. "Evaluation

of Cisplatin-Loaded Polymeric Micelles and

Hybrid Nanoparticles Containing Poly(Ethylene

Oxide)-Block-Poly(Methacrylic Acid) on Tumor

Delivery", Pharmacology \& Pharmacy, 2016.

Publication

\section{4}

Adel Mohamed Abd El-kader, Mahmoud Zaki El-

Readi, Amany Sayed Ahmed, Alaa Mohamed

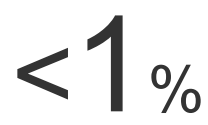

Nafady, Michael Wink, Zedan Zeid Ibraheim. "

Polyphenols from aerial parts of and their biological activities ", Pharmaceutical Biology, 2013

Publication

Exclude quotes 
Enhancing skin penetration of epigallocatechin gallate by modifying partition coefficient using reverse micelle method

GRADEMARK REPORT

FINAL GRADE

10

PAGE 1

PAGE 2

PAGE 3

PAGE 4

PAGE 5

PAGE 6

PAGE 7

PAGE 8

PAGE 9
GENERAL COMMENTS

Instructor 